\title{
SECOND-METACYCLIC FINITE $p$-GROUPS FOR ODD PRIMES
}

\author{
Vladimir Ćepulić, Olga Pyliavska and Elizabeta Kovač Striko \\ University of Zagreb, Croatia and National University Kyiv-Mohyla \\ Academy, Ukraine
}

\begin{abstract}
A second-metacyclic finite $p$-group is a finite $p$-group which possesses a nonmetacyclic maximal subgroup, but all its subgroups of index $p^{2}$ are metacyclic. In this article we determine up to isomorphism all second-metacyclic $p$-groups for odd primes $p$. There are ten such groups of order $p^{4}$, for each prime $p \geq 3$, and two such groups of order $3^{5}$.
\end{abstract}

\section{INTRODUCTION}

A second-metacyclic group is a group possessing a maximal nonmetacyclic subgroup, but it's second-maximal subgroups are all metacyclic. All secondmetacyclic finite 2-groups were determined in [1]. The aim of this article is to determine all second-metacyclic finite $p$-groups for $p>2$. We prove the following

THEOREM 1.1. Let $G$ be a second-metacyclic finite p-group for some odd prime $p$. Then either $|G|=p^{4}$, or $|G|=3^{5}$.

(1) If $|G|=p^{4}$, then $G$ is isomorphic to one of the following groups:

$G_{1}=\langle a, b, c, d| a^{p}=b^{p}=c^{p}=d^{p}=1,[a, b]=[a, c]=[a, d]=[b, c]$ $=[b, d]=[c, d]=1\rangle \cong E_{p^{4}}$,

$G_{2}=\left\langle a, b, c \mid a^{p^{2}}=b^{p}=c^{p}=1,[a, b]=[a, c]=[b, c]=1\right\rangle \cong Z_{p^{2}} \times E_{p^{2}}$,

$G_{3}=\langle a, b, c, d| a^{p}=b^{p}=c^{p}=d^{p}=1,[a, b]=[a, c]=[a, d]=[b, c]$ $=[b, d]=1,[c, d]=a\rangle=\langle b, c, d\rangle$, $Z\left(G_{3}\right)=\langle a, b\rangle, G_{3}^{\prime}=\langle a\rangle, \mho_{1}\left(G_{3}\right)=1$,

2000 Mathematics Subject Classification. 20D15.

Key words and phrases. Finite group, p-group, metacyclic, second-maximal subgroup, second-metacyclic subgroup. 


$$
\begin{aligned}
& G_{4}=\langle a, b, c, d| a^{p}=b^{p}=c^{p}=1, d^{p}=a,[a, b]=[a, c]=[a, d]=[b, c] \\
& =[b, d]=1,[c, d]=a\rangle=\langle b, c, d\rangle, \\
& Z\left(G_{4}\right)=\langle a, b\rangle, G_{4}^{\prime}=\langle a\rangle, \mho_{1}\left(G_{4}\right)=\langle a\rangle, \\
& G_{5}=\langle a, b, c, d| a^{p}=b^{p}=c^{p}=1, d^{p}=b,[a, b]=[a, c]=[a, d]=[b, c] \\
& =[b, d]=1,[c, d]=a\rangle=\langle b, c, d\rangle, \\
& Z\left(G_{5}\right)=\langle a, b\rangle, G_{5}^{\prime}=\langle a\rangle, \mho_{1}\left(G_{5}\right)=\langle b\rangle, \\
& G_{6}=\langle a, b, c, d| a^{p}=b^{p}=c^{p}=d^{p}=1,[a, b]=[a, c]=[a, d]=[b, c]=1, \\
& [b, d]=a,[c, d]=b\rangle=\langle c, d\rangle, \\
& Z\left(G_{6}\right)=\langle a\rangle, G_{6}^{\prime}=\langle a, b\rangle, \mho_{1}\left(G_{6}\right)=1, \\
& G_{7}=\langle a, b, c, d| a^{p}=b^{p}=c^{p}=1, d^{p}=a,[a, b]=[a, c]=[a, d]=[b, c]=1, \\
& [b, d]=a,[c, d]=b\rangle=\langle c, d\rangle, \\
& Z\left(G_{7}\right)=\langle a\rangle, G_{7}^{\prime}=\langle a, b\rangle, \mho_{1}\left(G_{7}\right)=\langle a\rangle, \\
& G_{8}=\langle a, b, c, d| a^{p}=b^{p}=c^{p}=1, d^{p}=a,[a, b]=[a, c]=[a, d]=[b, d] \\
& =[c, d]=1,[b, c]=a\rangle=\langle b, c, d\rangle, \\
& Z\left(G_{8}\right)=\langle d\rangle, G_{8}^{\prime}=\langle a\rangle, \mho_{1}\left(G_{8}\right)=\langle a\rangle, \\
& G_{9}=\langle a, b, c, d| a^{p}=b^{p}=c^{p}=1, d^{p}=a,[a, b]=[a, c]=[a, d]=[b, d]=1, \\
& [b, c]=a,[c, d]=b\rangle=\langle c, d\rangle, \\
& Z\left(G_{9}\right)=\langle a\rangle, G_{9}^{\prime}=\langle a, b\rangle, \mho_{1}\left(G_{9}\right)=\langle a\rangle, \\
& G_{10}=\langle a, b, c, d| a^{p}=b^{p}=c^{p}=1, d^{p}=a^{\sigma},[a, b]=[a, c]=[a, d]=[b, d]=1, \\
& [b, c]=a,[c, d]=b\rangle=\langle c, d\rangle,
\end{aligned}
$$

Here, the groups $G_{1}-G_{7}$ contain an elementary abelian subgroup $\langle a, b, c\rangle \cong E_{p^{3}}$, and the groups $G_{8}-G_{10}$ contain none such subgroup. For these groups $\langle a, b, c\rangle \cong S p\left(p^{3}\right)$, the special group of order $p^{3}$.

(2) If $|G|=3^{5}$, then $G$ is isomorphic to one of the following groups of maximal nilpotent class:

$$
\begin{aligned}
G_{11}= & \langle a, b, c, d, f| a^{3}=b^{3}=1, c^{3}=d^{3}=a, f^{3}=a b,[a, b]=[a, c]=[a, d] \\
& \left.=[a, f]=[b, c]=[b, f]=[c, f]=1,[b, d]=a^{2},[c, d]=b,[d, f]=c\right\rangle \\
= & \langle d, f\rangle, Z\left(G_{11}\right)=\langle a\rangle, Z_{2}\left(G_{11}\right)=\langle a, b\rangle, G_{11}^{\prime}=\langle a, b, c\rangle .
\end{aligned}
$$

Here, $M=\langle a, b, c, f\rangle$ is the unique abelian maximal subgroup of $G_{11}$.

$$
\begin{aligned}
G_{12}= & \langle a, b, c, d, f| a^{3}=b^{3}=1, c^{3}=d^{3}=a, f^{3}=b,[a, b]=[a, c]=[a, d] \\
& \left.=[a, f]=[b, c]=[b, f]=1,[b, d]=a^{2},[c, d]=b,[c, f]=a^{2},[d, f]=c\right\rangle \\
= & \langle d, f\rangle, Z\left(G_{12}\right)=\langle a\rangle, Z_{2}\left(G_{12}\right)=\langle a, b\rangle, G_{12}^{\prime}=\langle a, b, c\rangle .
\end{aligned}
$$

$G_{12}$ does not contain any abelian maximal subgroup. 


\section{Notation AND PRELIMINARIES}

At the beginning we recall some definitions and known results.

Definition 2.1. A group $G$ is metacyclic, $G \in \mathcal{M C}$, if it possesses a cyclic normal subgroup $N$ such that the factorgroup $G / N$ is also cyclic. We say that $G$ is proper metacyclic if $G$ is metacyclic but not cyclic.

Definition 2.2. $G$ is minimal nonmetacyclic, $G \in \mathcal{M C}_{1}$, if $G$ is not metacyclic, but all its maximal subgroups are metacyclic.

Definition 2.3. $G$ is second-metacyclic group, $G \in \mathcal{M C}_{2}$, if it possesses a nonmetacyclic maximal subgroup, but all its second-maximal subgroups are metacyclic.

Proposition 2.4. If $G$ is a proper metacyclic group of order $|G|=3^{3}$, then either

$$
\begin{gathered}
G=\left\langle x \mid x^{9}=1\right\rangle \times\left\langle y \mid y^{3}=1\right\rangle \cong Z_{9} \times Z_{3}, \text { or } \\
G=\left\langle x, y \mid x^{9}=y^{3}=1, x^{y}=x^{4}\right\rangle,
\end{gathered}
$$

for some $x, y \in G$, and it is $\mho_{1}(G)=\left\langle x^{3}\right\rangle, \Omega_{1}(G)=\left\langle x^{3}, y^{3}\right\rangle$ in both cases.

Proposition 2.5. If $G$ is a p-group of order $|G|=p^{2}$, then $G$ is abelian and $|A u t G|$, the order of its automorphism group, is divisible by $p$, but not by $p^{2}$.

Proposition 2.6. For $S \subseteq G$, denote by $\langle\langle S\rangle\rangle$ the normal closure of $S$ in $G$, that is the minimal normal subgroup of $G$ containing $S$. For $G=$ $\left\langle a_{1}, a_{2}, \ldots, a_{t}\right\rangle$ is $G^{\prime}=\left\langle\left\langle\left[a_{i}, a_{j}\right] \mid 1 \leq i<j \leq t\right\rangle\right\rangle$.

Theorem 2.7 (Blackburn [1], Huppert [3, III.14.2]). Let $G$ be a p-group of maximal class of order $p^{n}$. Then for each $k, 0 \leq k \leq n-2$, there exist exactly one normal subgroup $N$ of $G$ of order $p^{k}$ and $N=Z_{k}(G)=K_{n-k}(G)$.

Theorem 2.8 (Blackburn [1], Huppert [3, III.11.11]). Let G be a p-group, which is minimal nonmetacyclic. Then one of the following assertions holds:

(a) $G$ is elementary abelian of order $p^{3}$.

(b) It is $p>2$ and $G$ is nonabelian of exponent $p$ and order $p^{3}$.

(c) $G$ is a 3-group of class 3 and of order $3^{4}$.

(d) $G$ is a 2-group with $|G| \leq 2^{5}$.

Theorem 2.9 (Blackburn [1], Huppert [3, III.11.12]). Let $p>2$ and $|G|=p^{5}$, and let all subgroups of order $p^{3}$ in $G$ be generated by two elements. Then one of the following assertions holds:

(a) $G$ is metacyclic.

(b) $G$ is a 3-group of maximal class.

(c) The group $\Omega_{1}(G)$ is of order $p^{3}$ and exponent $p$ and $G / \Omega_{1}(G)$ is cyclic. 
THEOREM 2.10. If $G$ is a nonabelian p-group, possessing an abelian maximal subgroup, then $|G|=p \cdot\left|G^{\prime}\right| \cdot|Z(G)|$.

Proof. Let $A$ be a maximal subgroup of $G$ which is abelian, and $g \in$ $G \backslash A$. The mapping $\varphi: A \rightarrow A, \varphi(a)=[a, g]$, is homomorphism with $\operatorname{Im} \varphi=$ $G^{\prime}, \operatorname{Ker} \varphi=Z(G)$, and thus $A / Z(G) \cong G^{\prime}$.

Therefore $|A|=|G|: p=|Z(G)| \cdot\left|G^{\prime}\right|$ which yields to the above formula.

\section{Proof of Theorem 1.1}

Let $G \in \mathcal{M C}_{2}, G$ being a $p$-group for some odd prime $p$. By definition of $\mathcal{M C}_{2}$, the group $G$ contains some maximal subgroup $H \in \mathcal{M C}_{1}$, and by Theorem 2.8, $H$ is of order $p^{3}$ or $3^{4}$. Therefore $|G|=p^{4}$ or $|G|=3^{5}$.

In representing groups by generator orders and commutators, we shall omit, for brevity, those commutators of generators which equal 1 (that is for pairs of commuting generators).

A. CASE $|G|=p^{4}$.

Obviously, for $G^{\prime}=1, G$ must contain a subgroup $H$ isomorphic to $E_{p^{3}}$ and $\exp (G) \leq p^{2}$. Thus, in this case $G$ is isomorphic either to

$$
\begin{gathered}
G_{1}=\left\langle a, b, c, d \mid a^{p}=b^{p}=c^{p}=d^{p}=1\right\rangle \cong E_{p^{4}}, \text { or } \\
G_{2}=\left\langle a, b, c \mid a^{p^{2}}=b^{p}=c^{p}=1\right\rangle \cong Z_{p^{2}} \times E_{p^{2}} .
\end{gathered}
$$

In the following we assume that $G^{\prime}>1$. By Theorem 2.8 either $H^{\prime}=1$ and $H=\langle a, b, c\rangle \cong E_{p^{3}}$, or $H^{\prime} \neq 1$ and $H=\langle a, b, c| a^{p}=b^{p}=c^{p}=1,[b, c]=$ $a\rangle \cong S p\left(p^{3}\right)$.

A1. $H^{\prime}=1$ :

Now, $G=\left\langle H, d \mid d^{p} \in H\right\rangle$. By Theorem 2.10, $|G|=p \cdot\left|G^{\prime}\right| \cdot|Z(G)|=p^{4}$ and so either $|Z(G)|=p^{2},\left|G^{\prime}\right|=p$, or $|Z(G)|=p,\left|G^{\prime}\right|=p^{2}$.

$A 1.1|Z(G)|=p^{2},\left|G^{\prime}\right|=p$ :

Here, we may assume $G^{\prime}=\langle a\rangle<Z(G)=\langle a, b\rangle$, with $[c, d]=a$. Since $\left[d, d^{p}\right]=1$, it is $d^{p} \in Z(G)$ and we have, without loss, three different possibilities: $d^{p}=1, d^{p}=a^{\alpha} \neq 1$ and $d^{p}=b$. But if $d^{p}=a^{\alpha}$, then $\left[c^{\alpha}, d\right]=[c, d]^{\alpha}=a^{\alpha}$, as $[c, d] \in Z(G)$, and substituting $a^{\alpha}$ for $a$ and $c^{\alpha}$ for $c$, we get $[c, d]=d^{p}=a$ in this case. Thus we obtain three different $\mathcal{M C}_{2}$-groups:

$$
\begin{aligned}
& G_{3}=\left\langle H, d \mid d^{p}=1,[c, d]=a\right\rangle, \\
& G_{4}=\left\langle H, d \mid d^{p}=a,[c, d]=a\right\rangle, \\
& G_{5}=\left\langle H, d \mid d^{p}=b,[c, d]=a\right\rangle .
\end{aligned}
$$

$A 1.2\left|G^{\prime}\right|=p^{2},|Z(G)|=p:$

We may assume that $Z(G)=\langle a\rangle\left\langle G^{\prime}=\langle a, b\rangle\right.$. Denoting $\bar{x}=x\langle a\rangle$ for $x \in G$, we see that $(G /\langle a\rangle)^{\prime}=G^{\prime} /\langle a\rangle=\langle\bar{b}\rangle=Z(G /\langle a\rangle)$, as $|G /\langle a\rangle|=p^{3}$ and 
$G /\langle a\rangle$ is not abelian. Therefore, $b^{d}=a^{\alpha} b, \alpha \neq 0$ and $c^{d}=a^{\gamma} b^{\beta} c, \beta \neq 0$, as $b \in G^{\prime} \backslash Z(G)$. Substituting $a^{\alpha}$ for $a$ and $a^{\gamma} b^{\beta}$ for $b$, we get

$$
G=\left\langle a, b, c, d \mid d^{p} \in Z(G),[b, d]=a,[c, d]=b\right\rangle .
$$

We have two possibilities: $d^{p}=1$ or $d^{p}=a^{\delta}, \delta \neq 0$. If $d^{p}=1$, then we obtain

$$
G_{6}=\left\langle a, b, c, d \mid d^{p}=1,[b, d]=a,[c, d]=b\right\rangle .
$$

If $d^{p}=a^{\delta}$, then for $c^{\prime}=c^{\delta}, b^{\prime}=\left[c^{\prime}, d\right]=\left[c^{\delta}, d\right]=[c, d]^{\delta}=b^{\delta}, a^{\prime}=\left[b^{\prime}, d\right]=$ $\left[b^{\delta}, d\right]=[b, d]^{\delta}=a^{\delta}$, and we get, after substituting $a^{\prime}, b^{\prime}, c^{\prime}$ for $a, b, c$, the group

$$
G_{7}=\left\langle a, b, c, d \mid d^{p}=a,[b, d]=a,[c, d]=b\right\rangle .
$$

$A 2 . H \cong S p\left(p^{3}\right)$ and if $M<G$, then $M \nsubseteq E_{p^{3}}$ :

Now, $H=\left\langle a, b, c \mid a^{p}=b^{p}=c^{p}=1,[b, c]=a\right\rangle, Z(H)=\langle a\rangle$.

Let $K \leq H, K \triangleleft G,|K|=p^{2}$. Then $K \cong E_{p^{2}}$ and $N_{G}(K) / C_{G}(K)=$ $G / C_{G}(K) \leq A u t K$. We may assume without loss that $K=\langle a, b\rangle$.

As $p^{2} \chi|A u t K|$, by Proposition 2.5 it is $\left|G / C_{G}(K)\right| \leq p$ and there exists $A, A<G$, such that $K<A \leq C_{G}(K) \leq G$ and $|A|=p^{3}$. Since $K \leq Z(A), A$ is abelian. By assumption $A \nsubseteq E_{p^{3}}$ and also $A \nsubseteq Z_{p^{3}}$, as $G$ is not metacyclic. Thus $A \cong Z_{p^{2}} \times Z_{p}$. From $\langle a\rangle$ char $H \triangleleft G$ it follows $\langle a\rangle \triangleleft G$ and so $\langle a\rangle \leq Z(G)$. Therefore $a \in K$, since otherwise $\langle a\rangle \times K \cong E_{p^{3}}$, contradicting our assumption. Now, we may assume without loss that $K=\langle a, b\rangle=\Omega_{1}(A)$. Because of $\mho_{1}(A)$ char $A \triangleleft G$, it is $\mho_{1}(A) \leq Z(G)$ too. But $Z(H)=\langle a\rangle$ only, and so $\mho_{1}(A)=\langle a\rangle$. Thus $A=\left\langle a, b, d \mid d^{p}=a^{\alpha}, \alpha \neq 0\right\rangle$, for some $d \in A \backslash K$. The group $A$ is an abelian maximal subgroup in $G$, and by Theorem 2.10 it must be either $|Z(G)|=p^{2},\left|G^{\prime}\right|=p$, or $|Z(G)|=p,\left|G^{\prime}\right|=p^{2}$ again.

$A 2.1|Z(G)|=p^{2},\left|G^{\prime}\right|=p$ :

Now, $Z(G) \leq A$ because $G$ is not abelian, and we may assume without loss that $d \in Z(G)=\langle a, d\rangle=\langle d\rangle$. Let $\gamma$ be such that $\alpha \gamma \equiv 1(\bmod p)$. Then $\left(d^{\gamma}\right)^{p}=\left(d^{p}\right)^{\gamma}=a^{\alpha \gamma}=a$, and substituting $d$ by $d^{\gamma}$, we get the group

$$
G_{8}=\left\langle a, b, c, d \mid d^{p}=a,[b, c]=a\right\rangle .
$$

$A 2.2|Z(G)|=p,\left|G^{\prime}\right|=p^{2}$ :

Now, $Z(H)=\langle a\rangle=Z(G)<G^{\prime}$. Since $G / K$ is abelian, it is $G^{\prime} \leq K$ and so $G^{\prime}=K=\langle a, b\rangle$. Clearly, $[c, d] \in K \backslash\langle a\rangle$, as $\left.G^{\prime}\right\rangle\langle a\rangle$. Substituting $b^{\prime}=[c, d]$ for $b$, and $a^{\prime}=\left[b^{\prime}, c\right]$ for $a$, we get

$$
G=\left\langle a, b, c, d \mid a^{p}=b^{p}=c^{p}=1,[b, c]=a,[c, d]=b, d^{p}=a^{\alpha}\right\rangle,
$$

for some $\alpha \neq 1$. Substituting $c$ by $c^{\prime}=c^{\gamma}, \gamma \neq 0$, we get $\left[c^{\prime}, d\right]=\left[c^{\gamma}, d\right]=$ $b^{\gamma} a^{\varepsilon}=b^{\prime}$, for some $\varepsilon$, since $(G /\langle a\rangle)^{\prime}=\langle b, a\rangle /\langle a\rangle=Z(G /\langle a\rangle)$, and so $\left[c^{\gamma}, d\right] \equiv[c, d]^{\gamma} \equiv b^{\gamma}(\bmod \langle a\rangle)$. Now $\left[b^{\prime}, c^{\prime}\right]=\left[b^{\gamma} a^{\varepsilon}, c^{\gamma}\right]=\left[b^{\gamma}, c^{\gamma}\right]=a^{\gamma^{2}}=a^{\prime}$. Substituting $a^{\prime}, b^{\prime}, c^{\prime}$ for $a, b, c$ we get

$$
G=\left\langle a, b, c, d \mid a^{p}=b^{p}=c^{p}=1,[b, c]=a,[c, d]=b, d^{p}=a^{\frac{\alpha}{\gamma^{2}}}\right\rangle,
$$


with $\gamma \neq 0$, arbitrarily choosable. If $\alpha \equiv \tau^{2}(\bmod p)$, for some $\tau$, replacing $\gamma$ by $\tau$ we get

$$
G_{9}=\left\langle a, b, c, d \mid a^{p}=b^{p}=c^{p}=1,[b, c]=a,[c, d]=b, d^{p}=a\right\rangle .
$$

Otherwise, $\alpha \equiv \sigma \tau^{2}$, for some $\tau$ and, without loss, the minimal quadratic nonresidue $\sigma$ modulo $p$. Replacing again $\gamma$ by $\tau$ we get the group

$$
G_{10}=\left\langle a, b, c, d \mid a^{p}=b^{p}=c^{p}=1,[b, c]=a,[c, d]=b, d^{p}=a^{\sigma}\right\rangle,
$$

$\sigma$ being the minimal quadratic nonresidue modulo $p$.

B. CASE $|G|=3^{5}$.

Now, $G$ contains a maximal subgroup $H \in \mathcal{M C}_{1},|H|=3^{4}$. According to Theorem 2.8 the group $H$ is of maximal class. As $H$ is not metacyclic, its maximal subgroups cannot be cyclic. Thus, by Theorem 2.7 and Theorem 2.8, for each $L<H$ with $|L|=3^{3}$, it is $\exp (L)=3^{2}, \mho_{1}(L)=Z(H) \cong Z_{3}, \Omega_{1}(L)=$ $H^{\prime}=\Phi(H)=\Omega_{1}(H) \cong E_{9}$, since $\mho_{1}(L), \Omega_{1}(L) \triangleleft H$, as $L \triangleleft H$. We set $H^{\prime}=$ $\left\langle a, b \mid a^{3}=b^{3}=1\right\rangle, Z(H)=\langle a\rangle$ for some $a, b \in H$. Because of $\left|A u t E_{9}\right|=2^{4} \cdot 3$, we have $\left|H / C_{H}\left(H^{\prime}\right)\right|=3$, and the group $A=C_{H}\left(H^{\prime}\right)=\langle a, b, c\rangle$, where $c \in A \backslash$ $H^{\prime}$, is abelian. Let $K$ be an other maximal subgroup of $H, K=\langle a, b, d\rangle$. Now, $H=\langle A, K\rangle=\langle a, b, c, d\rangle$. If $K$ is abelian, then $\langle a, b\rangle \leq Z(H)$, a contradiction. Thus $K^{\prime}=Z(H)=\langle a\rangle$. We may assume without loss that $c^{3}=d^{3}=a$, as by Theorem 2.7 and Proposition 2.4, $\mho_{1}(K)=\langle a\rangle$ too. From $H=\langle\Phi(H), c, d\rangle$ and $[c, d] \in H^{\prime} \backslash Z(H)$, as $H^{\prime}>Z(H)$, it follows that we can set $[c, d]=b$ and $[b, d]=a^{\alpha}, \alpha \in\{1,2\}$. Now, $(c d)^{3}=c d^{3} c^{d^{2}} c^{d}=c a c b b a^{\alpha} c b=a^{\alpha+1} c^{3}=a^{\alpha+2}$. Since $c d \in H \backslash \Omega_{1}(H)$, it is $(c d)^{3} \neq 1$ implying $\alpha=2$. Therefore,

$$
H=\left\langle a, b, c, d \mid a^{3}=b^{3}=1, c^{3}=d^{3}=a,[b, d]=a^{2},[c, d]=b\right\rangle .
$$

We proceed to determine $G$. Since $|G|=3^{5}$ and all its subgroups of order $3^{3}$ are metacyclic, we can apply Theorem 2.9. As there is none subgroup of order $3^{3}$ and exponent 3 in $G$, it follows that $G$ is also of maximal class. Now, Theorem 2.7 implies that $Z(G)=Z(H)=\langle a\rangle, Z_{2}(G)=H^{\prime}, G^{\prime}=\Phi(G)=$ $C_{H}\left(H^{\prime}\right)=A$, as all these groups are characteristic in $H$, which is normal in $G$. Moreover, $\Omega_{1}(G)=H^{\prime}$, as $G \in \mathcal{M C}_{2}$ and $H^{\prime} \cong E_{9}$. Consider now, $M=C_{G}\left(H^{\prime}\right)=\langle a, b, c, f\rangle$, for some $f \in G \backslash H$. As $Z(M) \geq H^{\prime} \cong E_{9}$, and all maximal subgroups of $M$, being of order $3^{3}$, are metacyclic, $M$ must also be metacyclic, since otherwise it would be isomorphic to $H$, a contradiction because of $Z(H) \cong Z_{3}$. If $\exp (M)=3^{3}$, then we may choose $f$ such that $\langle f\rangle \cong Z_{27}$ and there is some $x \in\langle a, b\rangle \leq Z(M)$ such that $x \notin\langle f\rangle$ and therefore $M=\langle f\rangle \times\langle x\rangle \cong Z_{27} \times Z_{3}$. Now, $\mho_{1}(M)=\left\langle f^{3}\right\rangle \triangleleft G$, and $\left\langle f^{3}\right\rangle \cong Z_{9}$, which contradicts Theorem 2.7, as $Z_{2}(G) \cong E_{9}$. Therefore $\exp (M)=3^{2}$, and $M \cong\left\langle x, y \mid x^{9}=y^{9}=1,[x, y] \in\left\langle x^{3}\right\rangle\right\rangle$, for some $x, y \in M$. Now, $M^{\prime} \leq\left\langle x^{3}\right\rangle \cong Z_{3}$ and $M^{\prime} \triangleleft G$, which implies $M^{\prime} \leq\langle a\rangle$. Since $c^{3}=a$, it is $\langle c\rangle>M^{\prime}$ and so $\langle c\rangle \triangleleft M$. Therefore, we may assume $c=x, f=y$ and $\mho_{1}(M)=$ $\langle a, b\rangle$ implies $f^{3}=a^{\alpha} b^{\beta}$, with $\beta \in\{1,2\}$. Because of $G^{\prime}=\langle a, b, c\rangle=\Phi(G)$, 
it is $G=\langle a, b, c, d, f\rangle=\langle d, f\rangle$, and $G^{\prime}=\langle\langle[d, f]\rangle\rangle$ the normal closure of $\langle[d, f]\rangle$. Therefore $[d, f] \in A \backslash H^{\prime}$, since otherwise $G^{\prime}=H^{\prime}$, a contradiction. Substituting $f$ by $f^{2}$, if necessary, and substituting $c$ by $[d, f]$ we get again $[b, d]=a^{2}$ for these new generators. Moreover, $[c, f]=a^{\gamma}, \gamma \in\{0,1,2\}$ because $[c, f] \in M^{\prime} \leq\langle a\rangle$. Thus $G$ can be written as:

$$
\begin{array}{r}
G=\langle a, b, c, d, f| a^{3}=b^{3}=1, c^{3}=d^{3}=a, f^{3}=a^{\alpha} b^{\beta}, \\
\left.[d, f]=c,[c, d]=b,[c, f]=a^{\gamma},[b, d]=a^{2}\right\rangle .
\end{array}
$$

Now,

$$
\begin{aligned}
{\left[d, f^{3}\right] } & =[d, f]\left[d, f^{2}\right]^{f}=[d, f][d, f]^{f}[d, f]^{f^{2}} \\
& =c \cdot c^{f} \cdot c^{f^{2}}=c \cdot c a^{\gamma} \cdot c a^{\gamma} a^{\gamma}=c^{3}=a,
\end{aligned}
$$

and

$$
\begin{aligned}
{\left[d, f^{3}\right] } & =\left[d, a^{\alpha} b^{\beta}\right]=\left[d, b^{\beta}\right]=\left[b^{\beta}, d\right]^{-1} \\
& =\left(b^{-\beta}\left(b^{\beta}\right)^{d}\right)^{-1}=\left(b^{-\beta} b^{\beta} a^{2 \beta}\right)^{-1}=a^{\beta}
\end{aligned}
$$

implying $\beta=1$ and therefore $f^{3}=a^{\alpha} b$.

Next, we calculate $(d f)^{3}$ and $\left(d^{2} f\right)^{3}$, which should both be different from 1 :

$$
\begin{aligned}
(d f)^{3} & =d f^{3} d^{f^{2}} d^{f}=d a^{\alpha} b \cdot d c c a^{\gamma} \cdot d c=d^{2}\left(a^{\alpha} b\right)^{d} c^{2} a^{\gamma} d c \\
& =a^{\alpha+\gamma} d^{3}\left(a^{\alpha} b\right)^{d^{2}}\left(c^{2}\right)^{d} c=a^{\alpha+\gamma} \cdot a \cdot b a \cdot c^{2} b^{2} c=a^{\alpha+\gamma} a^{2} \cdot a=a^{\alpha+\gamma} \\
\left(d^{2} f\right)^{3} & =(d \cdot d f)^{3}=d(d f)^{3} d^{(d f)^{2}} d^{d f}=d a^{\alpha+\gamma} \cdot d c \cdot c a^{\gamma} b \cdot d c \\
& =a^{\alpha+2 \gamma} d^{3}\left(c^{2} b\right)^{d} c=a^{\alpha+2 \gamma+1} c^{2} b^{2} b a^{2} c=a^{\alpha+2 \gamma+1} .
\end{aligned}
$$

Therefore, $\alpha+\gamma \neq 0(\bmod 3), \alpha+2 \gamma+1 \neq 0(\bmod 3)$, which gives possibilities $(\alpha, \gamma) \in\{(1,0),(1,1),(0,2),(2,2)\}$. In the case $(\alpha, \gamma)=(1,0)$, $M^{\prime}=C_{G}\left(H^{\prime}\right)=\langle a, b, c, f\rangle^{\prime}=1$ and in other cases $M^{\prime}>1$. Replacing $f$ by $f^{2}, d$ by $d f, c$ by $c^{2}, b$ by $a^{2} b^{2}$, and $a$ by $a^{2}$, the case $(\alpha, \gamma)=(1,1)$ goes over in $(\alpha, \gamma)=(0,2)$, and replacing $d$ by $d f, c$ by $a^{2} c$ and $b$ by $a^{2} b$ the case $(\alpha, \gamma)=(2,2)$ goes over in $(\alpha, \gamma)=(0,2)$ as well. Thus there remain only the cases $(\alpha, \gamma)=(1,0)$ and $(\alpha, \gamma)=(0,2)$, giving the groups

$$
\begin{gathered}
G_{11}=\langle a, b, c, d, f| a^{3}=b^{3}=1, c^{3}=d^{3}=a, f^{3}=a b,[d, f]=c,[c, d]=b, \\
\left.[b, d]=a^{2}\right\rangle
\end{gathered}
$$

and

$$
\begin{gathered}
G_{12}=\langle a, b, c, d, f| a^{3}=b^{3}=1, c^{3}=d^{3}=a, f^{3}=b,[d, f]=c,[c, d]=b, \\
\left.[c, f]=a^{2},[b, d]=a^{2}\right\rangle .
\end{gathered}
$$

Our Theorem 1.1 is proved. 


\section{REFERENCES}

[1] N. Blackburn, Generalizations of certain elementary theorems on p-groups, Proc. London Math. Soc. (3) 11 (1961), 1-22.

[2] V. Ćepulić, M. Ivanković and E. Kovač Striko, Second-metacyclic finite 2-groups, Glas. Mat. Ser. III 40(60) (2005), 59-69.

[3] B. Huppert, Endliche Gruppen. I, Springer-Verlag, Berlin-New York, 1967.

V. Ćepulić

Department of Mathematics

Faculty of Electrical Engineering and Computing

University of Zagreb

Unska 3, HR-10000 Zagreb

Croatia

E-mail: vladimir.cepulic@fer.hr

O. Pyliavska

National University Kyiv-Mohyla Academy

Skorovody 2, Kyiv 04070

Ukraine

E. Kovač Striko

Faculty of Transport and Traffic Engineering

University of Zagreb

Vukelićeva 4, HR-10000 Zagreb

Croatia

E-mail: elizabeta.kovac@fpz.hr

Received: 28.9.2005. 